\title{
Study on the Vehicle Increase Prediction Model on Chinese Highways
}

\author{
Wei $\mathrm{Fu}$ \\ Department of Basic Course, Southwest Jiaotong University, Emei 614202, China \\ E-mail:wei_f730@163.com
}

\begin{abstract}
The vehicle increase prediction was discussed in the article. Starting from the actual situation and the characters of vehicle increase on the highways, various factors such as the large base of vehicle amount, the increase of average income, rural vehicle amount, the enhancement of comprehensive vehicle amount and the increase phenomena of vehicle purchase by young people to impact the vehicle increase on highways were considered comprehensively, and the statistical analysis were made aiming at these factors one by one, and the impacts of these factors on the vehicle increase on highways and the concrete impacting modes were discussed, and the vehicle increase model were established and improved. By the statistical software SPSS, the regression analysis of the curve parameter estimation was made to confirm the function form of the concrete curve, and the Mathematica and Matlab were used to confirm the parameters and influences of various factors in the model by a series of data fitting, and the final model were established.
\end{abstract}

Keywords: Statistical analysis, Curve fitting, Grey prediction, Differential equation

\section{Description of the problem}

Some new characters have occurred in the development of the vehicle on highways in recent years, and the increase of amount, the continual rise of the ratio of different vehicles, the average income and other factors all influence the increase of vehicle on highways. The "Report of National Vehicle Development Strategic Research" issued in the beginning of 2007 also further analyzed these characters.

There are many researches about the amount of vehicle, and abundant data materials have been accumulated.

In the article, starting from above characters about the actual situation of highway and the increase of vehicle, and using relative data (including searching relative literatures and supplementing new data) in the Appendix 2 for references, the mathematical model of the vehicle increase on highways is established, and the model is used to predict the middle and short-term trend and the long-term trend of the vehicle increase on highways.

\section{Hypotheses of the problem}

(1) Under normal situation, there are not large numerous of vehicles to enter and quit.

(2) The influence degrees of all discussed factors on the vehicles on highways can be denoted by the function about the time $t$, and they all change with the changes of $t$ according to certain rule.

(3) The ideal vehicle entering and quitting proportion is about 1:1, and this proportion will not influence the settlement of the problem.

(4) The ideal vehicle structure is the time structure in the stable vehicle model.

(5) Entering and quitting are independent each other.

(6) The speed of vehicle increase $v$ is proportional to the base amount of vehicle.

(7) The vehicle increase is limited by the total amount.

(8) The level of vehicle is higher, and the increase speed of vehicle amount is slower.

(9) It is generally to think that the unreasonable structure of vehicle increases the increase of vehicle on highways.

\section{Denotation of symbols}

$N$ is the total amount of vehicle at the time of $\mathrm{t}$.

$v$ is the vehicle increase speed at the time of $\mathrm{t}$.

$r$ is the vehicle increase rate at the time of $t$.

$\eta$ is the measurement value of vehicle quality at the time of $\mathrm{t}$. 
$N_{m}$ denotes the most total amount of vehicle which can be accepted by the highway.

$S$ denotes the difference between the vehicle structure and the expectation structure at the time of $\mathrm{t}$.

$W$ is the proportion of urban vehicle at the time of $t$.

$b$ is the vehicle increase rate at the time of $\mathrm{t}$.

$d$ is the vehicle scarp rate at the time of $\mathrm{t}$.

$\delta$ is the proportion of vehicle transfer at the time of $\mathrm{t}$.

\section{Analysis of the problem}

According to the statistical data of the traffic office, the core indexes influencing the vehicle increase on highway include the amount of vehicle possessed by the city along the highway, the vehicle structure (including truck and car), the vehicle speed, the speeds of vehicles with different structures, the work efficiency of the highway toll station, and the vehicle fault rate.

In recent years, the vehicle development of highway continually rise with the development of the society, the increase of vehicle speed, the continual increase of vehicle amount, the increase of rural vehicle, and the speed-raising of highway, and these factors all impact the amount of vehicle on highways.

Concretely speaking, the increase rate of highway vehicle amount is impacted by many factors, and the vehicle structure decides different speeds of vehicles, and the vehicle increase speed will be impacted to slow down, and the increase of vehicle speed will decrease the natural increase of vehicle amount, and the increase of vehicle fault rate will decrease the increase speed of the highway vehicle amount, and the increase of vehicle will accelerate the increase of the highway vehicle amount, and the vehicle structure will influence the increase rate of vehicle amount, i.e. the level of vehicle is higher and the fault rate is lower, which will make the natural increase rate of vehicle amount decrease, and the uncertain vehicle amount will accelerate the vehicle change, and the speed of vehicle increase is related with the base amount of highway vehicle, and the base amount of vehicle is larger, and the speed of vehicle increase is higher.

The classic model to describe the highway vehicle increase (Yang, 2003) is

$\left\{\begin{array}{l}v=\frac{d N}{d t}=\mathrm{r} \square\left(1-\frac{N}{N_{m}}\right) \square N \\ N\left(t_{0}\right)=N_{0}\end{array}\right.$

Where, $N_{m}$ denotes the most total amount of vehicle which can be accepted by the highway and $\mathrm{r}$ is the natural increase rate.

This model only considers the base amount of vehicle and the influence of limited resource to the vehicle increase, but doesn't consider the influences of vehicle speed, the continual increase of vehicle amount and the increase of urban vehicle on the highway vehicle increase, so we need improving this model to comprehensively consider these factors.

(1) Considering the influence of the base amount of vehicle, the speed of vehicle increase $v$ is proportional to the base amount of vehicle, i.e.

$v \propto N$

(2) Considering the factor of limited resource, the increase of vehicle is limited by the total amount of highway vehicle, and the mathematical description is

$v \propto\left(1-N / N_{\mathrm{m}}\right)$

(3) Considering the running speed of vehicle, i.e. the influence of the vehicle speed on the amount increase speed, the speed is higher and the vehicle increase speed is lower, i.e.

$v \propto 1 / \eta$

$\eta=\sum_{i} W_{i} \eta_{i}$

Where, $W_{i}$ denote the proportions of various types of vehicle. $\eta_{i}$ denotes the vehicle speed, and for the yearly value of $\eta_{i}, \eta_{i} \in\{0.25,0.50,0.75,1.0\}$, and for the type $\mathrm{C}$ vehicle speed, $\eta_{1}=0.25$, and for the type $\mathrm{B}$ vehicle speed, $\eta_{1}=0.50$, and for the type A vehicle speed, $\eta_{1}=0.75$, and for the vehicle speed with higher types, $\eta_{1}=1.0$.

(4) Considering the influence of amount structure on the vehicle increase speed, the reasonless vehicle structure will accelerate the increase of vehicle amount, i.e.

$v \propto S$

The so-called vehicle expectation structure means the stably increasing vehicle model in the vehicle increase model, and it can be denoted by the vehicle rate of each type. 
$S=\sum_{i}\left(b_{i}-a_{i}\right)^{2}$

Where, $a_{i}$ denotes the proportion of various vehicle amounts in the expectation time structure, $b_{i}$ denotes the rate of the $i$ 'th vehicle at the time of $t$.

Concretely, according to the mathematical knowledge of social vehicle, the static vehicle increase model, i.e. the age structure of stable model, is seen in Table 1.

(5) Considering the influence of village and town distribution on the vehicle increase speed, the town living condition is better than the condition of village, so the town vehicle will accelerate the increase of highway vehicle increase, i.e.

$v \propto W$

(6) Considering the influence of the vehicle type rate on the vehicle increase speed, the vehicle type rate is higher, and the increase of vehicle amount will be limited, so

$v \propto 1 /(1+S)$

(7) Considering the influence of entering rate and quitting rate of vehicle amount on the vehicle increase, the natural increase rate of vehicle amount equals to the different of the entering rate and the quitting rate, i.e.

$\mathrm{r}=\mathrm{b}-\mathrm{d}$

Where, the vehicle entering rate is

$b=\frac{\text { enteringam ount }}{\text { totalamoun } t \text { (highway) }}$

And it is influenced by the vehicle increase rate, and the entering rate from the city to the highway = entering amount/ the total amount of vehicle in the city. So,

$b=\frac{\text { enteringam ount }}{\text { totalcitya mount } * \text { totalamoun } t \text { (highway) }}$

At the same time, the vehicle increase speed is proportional to the natural increase rate of vehicle amount $\mathrm{r}$, so $v \propto r=b-d$

(8) Considering the influence of the proportion of fault vehicle amount in the vehicle amount on the vehicle increase, the fault proportion is higher, and the increase of vehicle amount is more limited, i.e. the increase speed is lower, $v \propto 1 / \delta$.

(9) The influence of flow vehicle amount on the vehicle increase speed, i.e. the increase of flow vehicle amount will add pressure to the highway, will accelerate the vehicle increase speed, and this factor and its influences are not considered in the article.

(10) Considering the influence of vehicle life on the vehicle increase, the enhancement of average vehicle life will increase the vehicle amount, and the vehicle increase will be impacted, and the vehicle increase speed will be accelerated.

So based on above factors influencing vehicle increase, the vehicle increase speed model can be established. Through data fitting by corresponding data, corresponding parameters can be solved, so the development trend of future vehicle amount can be predicted.

\section{Establishment of the model}

For the long-term prediction of highway vehicle increase, the model is established to predict this trend.

First, nine factors influencing the vehicle increase speed in the problem are changeable, i.e. these factors are the functions about the time $t$, and they can be denoted by

$f_{i}=f_{i}(t) ; i=1,2, . ., 9$

So, the model equation about the vehicle increase speed can be denoted by

Model1:

$$
\left\{\begin{array}{l}
v=\frac{d N}{d t}=K_{1} \prod_{i=1}^{9} f_{i}(t) \\
N\left(t_{0}\right)=N_{0}
\end{array}\right.
$$

Where, $\mathrm{K}_{1}$ is a constant number. 
Because the data can not allow us to quantitatively discuss the eighth and ninth factors (i.e. the proportion of fault vehicle amount in the total vehicle amount and the amount of flow vehicle), so the factors from the first factor to the seventh factor are used to establish the model.

Concretely, replace Model 1 by the concrete expressions of 7 factors, and the following model expression can be obtained.

Model2:

$$
\left\{\begin{array}{l}
v=\frac{d N}{d t}=\frac{K_{2} N W S r\left(1-N / N_{m}\right)}{\eta(1+\delta)(1+\varphi)} \\
N\left(t_{0}\right)=N_{0}
\end{array}\right.
$$

Where, $\mathrm{K}_{2}$ is a constant number, and

$$
\begin{aligned}
& N=N(t) ; S=S(t) ; \varphi=\varphi(t) ; \\
& r=r(t) ; W=W(t) ; \eta=\eta(t) \\
& \eta(t)=\sum_{i} W_{i} \sqcap \eta_{i} \\
& S(t)=\sum_{i}\left(b_{i}-a_{i}\right)^{2}
\end{aligned}
$$

According to relative data, the mathematical software is used to perform the data fitting to all functions about the time t, and the mathematical expressions about the time $t$ with good fitting effect are

$N=N(t) ; S=S(t) ; \varphi=\varphi(t) ; r=r(t) ; W=W(t) ; \delta=\delta(t) ; \eta=\eta(t)$

Put them into the original model equation, so

Model3:

$$
\left\{\begin{array}{l}
v=\frac{d N(t)}{d t}=\frac{K_{3} N(t) W(t) S(t) r(t)\left[1-N(t) / N_{m}\right]}{\eta(t)[1+\delta(t)][1+\varphi(t)]} \\
N\left(t_{0}\right)=N_{0}
\end{array}\right.
$$

Where, $\mathrm{K}_{3}$ is a constant number.

But, because the actual data are limited, the influences of the vehicle fault rate, the vehicle level and the flow vehicle amount on the vehicle increase speed are not analyzed, so these factors are not considered in the concrete problem. Modify the model 3 and eliminate $\delta, \eta$ and the flow vehicle amount, and the model 4 can be obtained.

Model4:

$$
\left\{\begin{array}{l}
v=\frac{d N(t)}{d t}=\frac{K N(t) W(t) S(t) r(t)\left[1-N(t) / N_{m}\right]}{[1+\varphi(t)]} \\
N\left(t_{0}\right)=N_{0}
\end{array}\right.
$$

Where, $\mathrm{K}$ is a constant number.

According to above formula, the mathematical software Mathematica is used to solve the vehicle amount function about the time $\mathrm{t}$ (year) (concrete program is seen in Appendix 3).

Model5:

$N=N(t)$

The expression is denoted by $t, K$ and $N$.

Replace the actual vehicle amount data $\left(t_{1}, N_{1}\right)$ and $\left(t_{2}, N_{2}\right)$ in $N=N(t)$, we can obtain the values of $\mathrm{K}$ and $\mathrm{N}$, so the expression of vehicle amount can be obtained.

$N=N(t)$

The problem is to predict the vehicle amount in future certain year, and to obtain the highway vehicle amount in future period, replace the time $t_{i}$ in the vehicle amount expression, so the predicted vehicle amount of that year can be obtained.

$N_{i}=N\left(t_{i}\right)$ 
By this way, we can obtain long-term prediction result.

For the middle-term and short-term prediction of highway vehicle increase, the $G M(1,1)$ model is used to perform the grey prediction.

(1) Associated coefficients.

Supposed that

$\hat{N}^{(0)}(k)=\left\{\hat{N}^{(0)}(1), \hat{N}^{(0)}(2), \ldots, \hat{N}^{(0)}(n)\right\} N^{(0)}(k)=\left\{N^{(0)}(1), N^{(0)}(2), \ldots, N^{(0)}(n)\right\}[0,1]$

So the associated coefficient is defined as

$\eta(k)=\frac{\min \min \left|\hat{N}^{(0)}(k)-N^{(0)}(k)\right|+\rho \max \max \left|\hat{N}^{(0)}(k)-N^{(0)}(k)\right|}{\left|\hat{N}^{(0)}(k)-N^{(0)}(k)\right|+\rho \max \max \left|\hat{N}^{(0)}(k)-N^{(0)}(k)\right|}$

Where, $\left|\hat{N}^{(0)}(k)-N^{(0)}(k)\right|$ is the absolute error between the k'th point $X^{(0)}$ and $\hat{X}^{(0)}$.

$\min \min \left|\hat{N}^{(0)}(k)-N^{(0)}(k)\right|$ is the minimum error between two classes.

$\max \max \left|\hat{N}^{(0)}(k)-N^{(0)}(k)\right|$ is the maximum error between two classes.

$\mathrm{p}$ is the distinguish-ability, $0<\rho<1$, generally, $\rho=0.5$.

For the sequences with different units and different initial values, they should be initialized before computing relative coefficients, i.e. eliminate the first value in all data in this sequence.

(2) Degree of association.

$r=\frac{1}{n} \sum_{k=1}^{n} \eta(k)$ is the association degree between $N^{(0)}(k)$ and $\hat{N}^{(0)}(k)$.

For the establishment of the $G M(1,1)$ model,

First, the time sequence $X^{(0)}$ has n observation values, $N^{(0)}=\left\{N^{(0)}(1), N^{(0)}(2), \ldots, N^{(0)}(n)\right\}$, and through accumulation, the new sequence $N^{(1)}=\left\{N^{(1)}(1), N^{(1)}(2), \ldots, N^{(1)}(n)\right\}$ generates, so the corresponding differential equation of the model $G M(1,1)$ is

$\frac{d N^{(1)}}{d t}+a N^{(1)}=\mu$

Where, $\alpha$ is the grey number of development, and $\mu$ is the endogenetic grey number.

Second, supposed $\hat{\alpha}$ is the solve-for parameter vector, $\hat{\alpha}=\left(\frac{a}{\mu}\right)$, it can be solved by the least square method.

$\hat{\alpha}=\left(B^{T} B\right)^{-1} B^{T} Y_{n}$

Solve the differential equation, and the prediction model can be obtained. 
Model6:

$\hat{N}^{(1)}(k+1)=\left[N^{(0)}(1)-\frac{\mu}{a}\right] e^{-a k}+\frac{\mu}{a}, k=0,1,2 \ldots, n$

Third, the test of grey prediction is the residual error test.

For the short-term and middle-term predictions of vehicle increase, the Model 5 can be used to solve these predictions.

\section{Solution of the problem}

(1) The grey prediction to the future short-term and middle-term vehicle amounts

According to the data, the statistics of the highway vehicle amount in recent years is seen in Table 2.

The corresponding scatter diagram is showed as follows.

FIGURE (simulation)

The grey prediction model of Model 6 is utilized to obtain the short-term vehicle amount by the Mathematica software, and the concrete situation is seen in Table 3 (concrete program is seen in Appendix).

(2) The model prediction to the long-term vehicle amount

First, solve the concrete expression of the model. To solve the function expressions of various factors about the time $t$, the data fitting should be implemented for corresponding data.

The least square method is used to perform the curve fitting of data.

Supposed the curve expression is $y=y(x)$.

Solve the objective function $\min z=\sum_{i}\left[y\left(x_{i}\right)-y_{i}\right]^{2}$, i.e. minimize $z$.

(1) The relationship fitting between the difference $\mathrm{S}$ of the age structure and the expectation age structure of vehicle amount at the time of $\mathrm{t}$ and the time of $\mathrm{t}$

By the software of EXCEL, the data in the appendix (including data from Internet) are processed to table the corresponding values of $\mathrm{s}$ and $\mathrm{t}$ (seen in Table 4).

From the data in the table, (supposed) on abnormal point $(11,0.0027)$ is found, the data fitting at this point should not be performed. Perform the relationship fitting of $\mathrm{S}$ and $\mathrm{t}$ by the data. First, the future vehicle amount fault rate will be reduced gradually according to the prediction information (seen in Figure 1), and the reasonless speed of vehicle structure will be reduced also, so one function curve corresponding with the curve in the following figure is tried to find to perform the data fitting.

Figure 1. Prediction Diagram

For the data analysis, the software SPSS is used to perform the multiple regression analysis about the curve parameter estimation, and the information obtained is seen in Table 5.

From the information in Table 5, through repetitive fitting and analysis of curve, the function form with good fitting curve with the original data, i.e. the power function form can be found.

For the parameter estimation, perform the power function curve fitting to the data, so

$S(t)=0.0000039666 t^{3}-0.023714 t^{2}+47.259 t-31393$

(2) The relationship fitting between the difference $\varphi$ of the vehicle type rate and normal value in the year $\mathrm{t}$ and the time of $\mathrm{t}$

In the same way of (1), the corresponding values of $\varphi$ and $t$ are seen in Table 6.

According to the prediction information in appendix (seen in Figure 2), the general development trend of the future vehicle type rate can be obtained, so one curve basically corresponding with the curve in the following figure can be found to perform the data fitting.

Figure 2. Variance of New-added Vehicle Type Rate Period

In the same ways of (1), the SPSS is used to perform the regression analysis to the data, the fitting function form of the optimal fitting curve, i.e. trigonometric function, can be obtained, and then use the Matlab to perform the curve fitting of data. By the data fitting, the following formula can be obtained.

$\varphi(t)=0.0152104 \operatorname{Sin}(81.5969+0.960176 \mathrm{x})$

(3) The relationship fitting of the natural increase rate $r$ of the vehicle amount and the time of $t$ in the t'th month 
As seen in Table 7, the quitting rate can be computed according to the relative data.

Part result of data processing is seen in Table 8.

So the data about the corresponding natural increase rate $\mathrm{r}$ can be obtained.

In the same way of (1), combining with the entering rate and the quitting rate, the corresponding values of $r$ and $t$ are seen in Table 9.

In the same way of (1), the SPSS is used to perform the regression analysis for the data, and the fitting function form of the optimal fitting curve can be obtained, and then the Matlab is used to perform the curve fitting for data.

Through data fitting, the following formula can be obtained.

$r(t)=0.00048983 t^{3}-2.9258 t^{2}+5824.8 t-3864900$

(4) The relationship fitting between the urban vehicle amount proportion and the time of $t$ in the year of $t$

In the same way of (1), combining with the entering rate and the quitting rate, the corresponding values of $\mathrm{W}$ and $\mathrm{t}$ are seen in Table 10 .

In the same way of (1), the SPSS is used to perform the regression analysis for the data, and the fitting function form of the optimal fitting curve can be obtained, and then the Matlab is used to perform the curve fitting for data.

Through the data fitting, the following formula can be obtained.

$W(t)=-0.000060889 t^{3}+0.36533 t^{2}-730.66 t+487090$

The relationships between four factors including the vehicle amount age structure, the natural increase rate, the type property, and the urban vehicle amount proportion with the time $t$ are found through above analysis and computations, i.e.

$S=S(t) ; \quad \varphi=\varphi(t)$

$r=r(t) ; \quad W=W(t)$

According to the model 4, replace the concrete expressions of $s=s(t) ; \varphi=\varphi(t) ; r=r(t) ; W=W(t)$, and $N\left(t_{0}=2005\right)=1308$ (supposed that the total vehicle amount is 13.08 million in January of certain year) in the model 4 , the differential equation about the time $\mathrm{t}$ can be obtained.

$$
\left\{\begin{array}{l}
\frac{d N(t)}{d t}=\frac{K N(t) W(t) S(t) r(t)\left[1-N(t) / N_{m}\right]}{[1+\varphi(t)]} \\
N\left(t_{0}=2005\right)=1308 \\
S(t)=0.0000039666 t^{3}-0.023714 t^{2}+47.259 t-31393 \\
r(t)=0.00048983 t^{3}-2.9258 t^{2}+5824.8 t-3864900 \\
W(t)=-0.000060889 t^{3}+0.36533 t^{2}-730.66 t+487090 \\
\varphi(t)=0.0152104 \operatorname{Sin}(81.5969+0.960176 \mathrm{t})
\end{array}\right.
$$

Utilize the software Mathematica to solve the differential equation, and the expression about $t, K$ and $N_{m}$ of the vehicle amount can be obtained (program and result are seen in appendix 4). Replace any two groups of actual vehicle amount data $\left(t_{1}, N_{1}\right)$ and $\left(t_{2}, N_{2}\right)$ in $N=N(t)$, and use the software of Matlab to solve the equation group with two unknowns, and $K=-1.1 e^{-6}, N_{m}=81$. So the expression of vehicle amount can be obtained.

$$
N(t)=\frac{810000}{1+\frac{1}{130756} e^{\left(-1.2 \times 10^{-11} t^{7}-1.6228 \times 10^{-7} t^{6}-0.00097 t^{5}+3.23082 t^{4}-6446.693 t^{3}+7717896 t^{2}-5133023692 t\right)}}
$$

Next, this model is used to predict the development of vehicle amount in future 50 years in China.

Replace the time (year) in the expression, and obtain the predicted vehicle amount.

$N_{i}=N(i) ; i=2006,2007, . ., 2055$

$N_{i}$ denotes the total amount of vehicle in the years of $i$, and the total vehicle amount in china from 2006 to 2055 can be predicted.

The software Matlab can be used to seek the total amount of vehicle in China from 2006 to 2055.

The mathematical software is used to draw the long-term vehicle increase line chare on highways. 


\section{Evaluation and extension of the model}

This model comprehensively considers the influences of numerous factors on the vehicle increase speed in China, and a comprehensively general mathematical model about the vehicle increase is established. The character of this model is to take the function model of vehicle amount about the time of $\mathrm{t}$ as the base, and respectively establish function relationships about the time of $t$ under the influences of many factors such as vehicle type proportion, vehicle amount age structure, vehicle amount comprehensive quality, and the village and city vehicle amount distribution. These factors were comprehensively considered to establish the vehicle amount prediction model to perform the prediction. By this way, the analysis of single variable will reduce the complexity of the problem and make the establishment of the model become more easily. At the same time, in the settlement of the model, the fitting precision is considered fully, so the final model is more reasonable, and the prediction result of the vehicle amount is more reasonable.

There are deficiencies in the model. For the short-term and middle-term vehicle amount prediction, only the classic grey prediction method was used, but the grey prediction can only predict the short-term vehicle increase, and it will produce large prediction error for the long-term vehicle amount. So we attempted to use the prediction result of the first time to predict the result of the second time, but the prediction effect was not good, so for the middle-term vehicle amount prediction, the method is not efficient enough. At the same time, the rationality and the validity of the model will depend on the fitting precision and quality of a series of data, which would bring difficulties to establish the reasonable model and prediction.

In addition, for part problems in the vehicle amount model, the probability statistics knowledge can be used to establish the model, and the normal distribution can be used to establish and solve the model.

This model is the vehicle amount prediction model, and this model can predict the vehicle amount on highways in future, which can help the traffic office to control the vehicle amount and eliminate the burst fuse of the vehicle amount on highways.

\section{References}

Jiang, Qiyuan et al. (2003). Mathematical Model (3rd edition). Beijing: Higher Education Press. Aug of 2003.

Tan, Yongji et al. (2004). Mathematical Model. Shanghai: Fudan University Press. Aug of 2004.

Xiao, Wei et al. (2005). Matlab Program Design and Application. Beijing: Tsinghua University Press \& Beijing Jiaotong University Press. July of 2003.

Yang, Mingsheng et al. (2003). Mathematica Base and Mathematical Software. Dalian: Dalian University of Technology Press. Aug of 2003.

Table 1. Age structure of the quiet vehicle increase mode

\begin{tabular}{|c|c|c|c|c|}
\hline Vehicle type & A & B & C & Senior vehicle \\
\hline Proportion & 0.25 & 0.5 & 0.2 & 0.05 \\
\hline
\end{tabular}

Table 2. Data statistics of vehicle amount (unit: million)

\begin{tabular}{|c|c|c|c|c|c|c|c|c|c|c|}
\hline Month & 1 & 2 & 3 & 4 & 5 & 6 & 7 & 8 & 9 & 10 \\
\hline Amount of vehicle & 1224 & 1236 & 1248 & 1258 & 1267 & 1276 & 1285 & 1292 & 1300 & 1308 \\
\hline
\end{tabular}

Table 3. Short-term prediction of vehicle amount (unit: million)

\begin{tabular}{|c|c|c|c|c|c|c|c|c|c|c|}
\hline Year & 11 & 12 & 13 & 14 & 15 & 16 & 17 & 18 & 19 & 20 \\
\hline Amount of vehicle & 1319 & 1328 & 1337 & 1346 & 1356 & 1365 & 1375 & 1384 & 1394 & 1403 \\
\hline
\end{tabular}

Table 4. Corresponding values of $\mathrm{S}$ and $\mathrm{t}$

\begin{tabular}{|c|c|c|c|c|c|c|c|c|}
\hline $\mathrm{t}$ (month) & 16 & 15 & 14 & 13 & 12 & 11 & 10 & 9 \\
\hline $\mathrm{S}(\mathrm{t})$ & 0.0638 & 0.0624 & 0.0607 & 0.0592 & 0.0577 & 0.0027 & 0.0553 & 0.0551 \\
\hline $\mathrm{T}($ month) & 8 & 7 & 6 & 5 & 4 & 3 & 2 & 1 \\
\hline $\mathrm{S}(\mathrm{t})$ & 0.0548 & 0.0545 & 0.0543 & 0.0541 & 0.0538 & 0.0536 & 0.0535 & 0.0534 \\
\hline
\end{tabular}


Table 5. Model summary and parameter estimates

Dependent Variable: VAR00002

\begin{tabular}{|c|c|c|c|c|c|c|c|c|c|}
\hline \multirow[b]{2}{*}{ Equation } & \multicolumn{5}{|c|}{ Model Summary } & \multicolumn{4}{|c|}{ Parameter Estimates } \\
\hline & R Square & $\mathrm{F}$ & $d f 1$ & $\mathrm{df} 2$ & Sig. & Constant & b1 & $\mathrm{b} 2$ & b.3 \\
\hline Linear & .154 & 1.080 & 1 & 6 & .337 & 6.424 & .003 & & \\
\hline Logarithmid & .154 & 1.089 & 1 & 6 & .337 & -49.168 & 6.475 & & \\
\hline Quadratic & .154 & 1.080 & 1 & 6 & .337 & -3.187 & .000 & OBE-007 & \\
\hline Cubic & .154 & 1.091 & 1 & 6 & .336 & -2.108 & .000 & .000 & 69E-010 \\
\hline Compound & .086 & .566 & 1 & 6 & .480 & $35 E-115$ & 1.139 & & \\
\hline Power & .086 & .566 & 1 & 6 & .481 & .000 & 261.173 & & \\
\hline$S$ & .086 & .565 & 1 & 6 & .481 & 257.849 & -522776 & & \\
\hline Growth & .086 & .566 & 1 & 6 & .480 & -264.497 & .130 & & \\
\hline Exponentia & .086 & .566 & 1 & 6 & .480 & $35 E-115$ & .130 & & \\
\hline
\end{tabular}

The independent variable is VARO0001.

Table 6. Corresponding values of the vehicle type rate warp and the time $t$

\begin{tabular}{|c|c|c|c|c|c|c|c|c|}
\hline $\mathrm{t}$ (month) & 15 & 14 & 13 & 12 & 11 & 10 & 9 & 8 \\
\hline$\varphi_{(\mathrm{t})}$ & 0.0630 & 0.0629 & 0.0620 & 0.0606 & 0.0600 & 0.0674 & 0.0589 & 0.0513 \\
\hline $\mathrm{t}($ year) & 7 & 6 & 5 & 4 & 3 & 2 & 1 & 0 \\
\hline$\varphi_{(\mathrm{t})}$ & 0.0436 & 0.033411 & 0.042065 & 0.045082 & 0.041812 & 0.04273 & 0.055166 & 0.062693 \\
\hline
\end{tabular}

Table 7. Fitting of the relationship between the natural increase rate of vehicle amount in the $t$ 'th month with the time $t$

\begin{tabular}{|c|c|c|c|c|c|c|c|c|}
\hline Unit: \% & 8 & 9 & 10 & 11 & 12 & 13 & 14 & 15 \\
\hline Entering rate b & 15.64 & 14.64 & 14.03 & 13.38 & 12.86 & 12.41 & 12.29 & 12.4 \\
\hline
\end{tabular}

Table 8. Part result of data processing

\begin{tabular}{|c|c|c|c|c|c|c|c|c|}
\hline Unit: \%o & 8 & 9 & 10 & 11 & 12 & 13 & 4 & 15 \\
\hline Quitting rate b & 6.5 & 6.46 & 6.45 & 6.43 & 6.41 & 6.4 & 6.42 & 6.51 \\
\hline
\end{tabular}

Table 9. Corresponding values of $\mathrm{r}$ and $\mathrm{t}$

\begin{tabular}{|c|c|c|c|c|c|c|c|c|}
\hline $\mathrm{t}$ (month) & 15 & 14 & 13 & 12 & 11 & 10 & 9 & 8 \\
\hline $\mathrm{r}(\mathrm{t})$ & 5.89 & 5.87 & 6.01 & 6.45 & 6.95 & 7.58 & 8.18 & 9.14 \\
\hline $\mathrm{t}($ month) & 7 & 6 & 5 & 4 & 3 & 2 & 1 & 0 \\
\hline $\mathrm{r}(\mathrm{t})$ & 10.06 & 10.42 & 10.55 & 11.21 & 11.45 & 11.6 & 12.98 & 14.39 \\
\hline
\end{tabular}

Table 10. Corresponding values of $\mathrm{W}$ and $\mathrm{t}$

\begin{tabular}{|c|c|c|c|c|c|c|c|c|}
\hline $\mathrm{t}$ (month) & 1 & 2 & 3 & 4 & 5 & 6 & 7 & 8 \\
\hline $\mathrm{W}(\mathrm{t})$ & $26.94 \%$ & $27.46 \%$ & $27.99 \%$ & $28.51 \%$ & $29.04 \%$ & $30.48 \%$ & $31.91 \%$ & $33.35 \%$ \\
\hline $\mathrm{t}$ (month) & 9 & 10 & 11 & 12 & 13 & 14 & 15 & \\
\hline $\mathrm{W}(\mathrm{t})$ & $34.78 \%$ & $36.22 \%$ & $37.66 \%$ & $39.09 \%$ & $40.53 \%$ & $41.76 \%$ & $42.99 \%$ & \\
\hline
\end{tabular}

